\title{
Kazhdan constants of group extensions
}

\author{
Uzy Hadad
}

February 6, 2020

\begin{abstract}
We give bounds on Kazhdan constants of abelian extensions of (finite) groups. As a corollary, we improved known results of Kazhdan constants for some meta-abelian groups and for the relatively free group in the variety of $p$-groups of lower $p$-series of class 2 . Furthermore, we calculate Kazhdan constants of the tame automorphism groups of the free nilpotent groups.
\end{abstract}

\section{Introduction.}

Property (T) was introduced by Kazhdan $\mathrm{Kaz}$ in 1967. It founds numerous applications in various areas of mathematics, in particular for estimating quantitative behavior of the mixing time of random walks and the expansion of the Cayley graphs of finite groups. For introduction to the subject and applications we refer the reader to $[\mathrm{BHV}, \mathrm{Lub}]$.

Definition 1.1. Let $\Gamma$ be a discrete group, $S \subset \Gamma$ a subset, $\epsilon>0$, and let $\left(\rho, \mathcal{H}_{\rho}\right)$ be a unitary representation of $\Gamma$. A vector $0 \neq v \in \mathcal{H}_{\rho}$ is called $(S, \epsilon)$-invariant, if for every $s \in S$, we have $\|\rho(s) v-v\| \leq \epsilon\|v\|$.

Definition 1.2. Let $\Gamma$ be a discrete group and let $S$ be a finite generating subset.

1. The group $\Gamma$ is said to have Kazhdan property $(T)$ if there exists an $\epsilon>0$ such that every unitary representation with $(S, \epsilon)$-invariant vector, contains a non-zero $\Gamma$-invariant vector. In that case $\epsilon$ is called a Kazhdan constant of $\Gamma$ with respect to $S$.

2. More generally, if $\mathcal{C}$ is a class of unitary representations of $\Gamma$, we say that $\epsilon$ is a Kazhdan constant of $\Gamma$, with respect to the set $S$ and relative to the class $\mathcal{C}$, if every representation in $\mathcal{C}$ with $(S, \epsilon)$-invariant vector, contains a non-zero $\Gamma$-invariant vector.

It turns out (see subsection 1.2) that in case of a finite group, it is more appropriate to define a Kazhdan constant of a group with respect to the mean instead of the maximum over the generators, thus, we give the following definition: 
Definition 1.3. Let $\Gamma$ be a discrete group and let $S$ be a finite generating multiset of $\Gamma$.

1. The group $\Gamma$ has Kazhdan property $(T)$ if there exist an $\epsilon>0$ s.t

$$
\inf _{\rho \in \Gamma_{0}^{*}} \inf _{0 \neq v \in \mathcal{H}_{\rho}} \frac{1}{|S|} \sum_{s \in S} \frac{\|\rho(s) v-v\|^{2}}{\|v\|^{2}} \geq \epsilon,
$$

where $\Gamma_{0}^{*}$ denote the space of unitary representations of $\Gamma$ with no invariant non-zero vectors. In that case $\epsilon$ is called an average Kazhdan constant of $\Gamma$ with respect to $S$.

2. More generally, if $\mathcal{C}$ is a class of unitary representations of $\Gamma$ with no invariant non-zero vectors, then $\epsilon>0$ is called an average Kazhdan constant of $\Gamma$, with respect to the set $S$ and relative to the class $\mathcal{C}$, if

$$
\inf _{\rho \in \mathcal{C}} \inf _{0 \neq v \in \mathcal{H}_{\rho}} \frac{1}{|S|} \sum_{s \in S} \frac{\|\rho(s) v-v\|^{2}}{\|v\|^{2}} \geq \epsilon .
$$

This definition is equivalent to the previous definition, they only differ in $\epsilon$. One can check that the infimum in the average Kazhdan constant is obtained at an irreducible representation.

In general, for a group $\Gamma$ with a Kazhdan constant $\epsilon_{1}$ with respect to $S$, and with an average Kazhdan constant $\epsilon_{2}$ (w.r.t $S$ ), we have the following inequalities $\epsilon_{1}^{2} \geq \epsilon_{2} \geq \frac{\epsilon_{1}^{2}}{|S|}$.

Throughout this paper the notation Kazhdan constant will be used for the case of infinite groups and an average Kazhdan constant for finite groups.

For estimations of Kazhdan constants for various groups see [Bag, BH, Bur, Sh1, Kas2].

Let $\Gamma$ be a finitely generated group with a Kazhdan constant $\epsilon_{1}$ with respect to $S_{1}$. Let $S_{2}$ be another generating set then it is not hard to prove (see [Lub], rem. 3.2.5) that there exists $\epsilon_{2}=\epsilon_{2}\left(\epsilon_{1}, S_{1}, S_{2}\right)$ such that the Kazhdan constant of $\Gamma$ with respect to $S_{2}$ is greater than or equal to $\epsilon_{2}$. For the interesting question of the dependance of Kazhdan constants on the generating sets, see [ALW, GZ, Kas1]. This bring us to the following definition:

Definition 1.4. Let $\Gamma$ be a finite group. Given $0<\epsilon<1$, we define $g_{\epsilon}(\Gamma)$ as the minimal integer $N$, such that there is a generating set $S$ of $\Gamma$, with $|S| \leq N$, and the average Kazhdan constant of $\Gamma$ with respect to $S$ is greater than or equal to $\epsilon$.

The number $g_{\epsilon}(\Gamma)$ is important in many theoretical and practical computational problems, hence we ask the following natural question.

Question 1.5. For a finite group $\Gamma$ and $0<\epsilon<1$, what is $g_{\epsilon}(\Gamma)$ ?

N. Alon and Y. Roichman (Corollary 1 in [AR], see also [LR, LS]) showed that $g_{\epsilon}(\Gamma) \leq$ $O_{\epsilon}(\log |\Gamma|)$ for any $0<\epsilon<1$. This bound is sharp for the case of abelian groups. However, 
for any finite non-abelian simple group $\Gamma$ (except Suzuki), it has been proved recently by Kassabov, Lubotzky and Nikolov [KLN], that $g_{\epsilon}(\Gamma)=O(1)$ for some $0<\epsilon<1$.

One of the goals of this paper is to present a method for estimating $g_{\epsilon}(\Gamma)$ for some nonsimple groups.

\subsection{Main Results}

Theorem 1.6. Let $A, H$ be two finite groups with $A$ abelian, such that $H$ acts on $A$. Let $\Gamma$ be an extension of $A$ by $H$. Let $S \subset H, \tilde{S}$ a lifting of the set $S$ to $\Gamma, B \subset A$ and $\tilde{B}=\left\{a^{x} \mid a \in B, x \in H\right\}$ be the union of the orbits. Assume that the following hold:

1. $H$ is generated by $S$ and has average Kazhdan constants $\epsilon_{H}$ with respect to $S$.

2. $A$ is generated by $\tilde{B}$ and has average Kazhdan constants $\epsilon_{A}$ with respect to $\tilde{B}$.

Then the group $\Gamma$ has average Kazhdan constants $\epsilon>\frac{\epsilon_{H} \epsilon_{A}}{512 \cdot\left(1+\frac{|S|}{|B|}+\frac{|B|}{|S|}\right)}$ with respect to the set of generators $\tilde{S} \cup B$.

Note that the conclusion of the theorem holds for an arbitrary lifting $\tilde{S}$ of $S$, with the same constant. Moreover, the assumptions on $A$ are with respect to the generating set $\tilde{B}$ which may be very large (of size $|B| \cdot|H|$ ) whereas the generating set of $\Gamma$ might be much smaller.

This Theorem is an extension of a work by Alon, Lubotzky and Wigderson [ALW], who proved a similar result for the the case of semi-direct product.

The main idea of the proof of Theorem 1.6 is the connection between almost $\Gamma$-invariant vector and almost $H$-invariant measure on the unitary dual of $A$. If there exists an almost invariant vector with respect to the generating set of $\Gamma$, then one can associate with it a measure on the unitary dual of the abelian group $A$. We show that this measure is almost $H$-invariant under the dual action of $H$ on $\hat{A}$, and this brings to a contradiction.

Our method is generic and makes no assumption on the finite groups $A$ and $H$. However, for some specific groups (even infinite) $A$ and $H$, one can do better. For example, see Bur, Kas1, Sh1], where the authors study the group $E L_{n}(R) \ltimes R^{n}$ (where $R$ is an associative ring) with respect to some unipotent matrices as a generating set.

The following is deduced from the main Theorem:

Corollary 1.7. Let $n \in \mathbb{N}, p$ a prime, and let $C_{p}, C_{p^{n}}$ be the cyclic groups of order $p$ and $p^{n}$ respectively. Let $\Gamma$ be any extension of the group algebra $\mathbb{F}_{p}\left[C_{p}\right]$ by $C_{p^{n}}$, where $C_{p^{n}}$ acts by left translation $(\bmod p)$ on $\mathbb{F}_{p}\left[C_{p}\right]$. Then there exists $\epsilon>0$, such that $g_{\epsilon}(\Gamma) \leq O_{\epsilon}(n \log p)$.

Note that by Alon-Roichman Theorem, $g_{\epsilon}(\Gamma) \leq O_{\epsilon}((n+p) \log p)$.

Theorem 1.6 covers the case of non-trivial extensions. For the case of central extension the following is a quantitative version of a result of Serre. 
Theorem 1.8 (Serre). Let $\Gamma$ be a central extension of $A$ by $H$, i.e $A \subseteq \boldsymbol{Z}(\Gamma)$. Let $\tilde{S} \subset \Gamma$ be a set of generators of $\Gamma$ and let $S$ be the projection of $\tilde{S}$ to $H$. Assume that $\epsilon_{1}$ (resp. $\epsilon_{2}$ ) is a (resp. an average) Kazhdan constant of $H$ with respect to $S$. Then $\frac{\epsilon_{1}}{2 \cdot|S|}$ (resp. $\frac{\epsilon_{2}}{4}$ ) is a (resp. an average) Kazhdan constant of $\Gamma$ with respect to $\tilde{S}$ with respect to any unitary representation of $\Gamma$ which does not contains a one dimensional subrepresentation.

For the proof we refer the reader to $[\mathrm{HV}]$ (pages $26-28$ or Lemma 1.7 .10 in [BHV]). Although the quantitative result is not stated there explicitly, it follows immediately from the proof. In subsection 3.4 we use this result for estimating Kazhdan constants of the tame automorphism groups of the free nilpotent groups, extending a result of Mallahi [MAK]. These estimations have applications for evaluating the mixing time of the product replacement algorithm.

To illustrate the use of the above result for concrete groups, we prove the following:

Corollary 1.9. For a fixed prime $p$, let $\Gamma_{k, 2}$ be the relatively free group on $k$ generators in the variety of $p$-groups of lower $p$-series of class 2 , then for every $0<\epsilon<\frac{1}{4}$ we have $g_{\epsilon}\left(\Gamma_{k, 2}\right) \leq O_{\epsilon}\left(\sqrt{\log \left|\Gamma_{k, 2}\right|}\right)$.

So our method improves Alon-Roichamn result which require $O_{\epsilon}\left(\log \left|\Gamma_{k, 2}\right|\right)$ elements. For the definition and properties of the group $\Gamma_{k, 2}$ see subsection 3.3 .

\subsection{The Spectral Gap}

Let $G=\operatorname{Cay}(\Gamma, S)$ be the Cayley graph of a finite group $\Gamma$ with respect to a symmetric generating set $S\left(S=S^{-1}\right)$. Let $A_{r}$ be the normalized adjacency matrix of the Cayley graph $G$. We denote by $\lambda(\Gamma, S)$ the second largest eigenvalue of $A_{r}$ and we also define its spectral gap $\beta=1-\lambda(\Gamma, S)$ (for application of $\beta$ see [HLW]). It turn out that there is a tight connection between the average Kazhdan constant and the spectral gap of $G$, indeed we have (see fact 1.1 in [MW] and section 11 in [HLW]):

$$
\beta=\inf _{\rho \in \Gamma_{0}^{*}} \inf _{0 \neq v \in \mathcal{H} \rho} \frac{1}{2|S|} \sum_{s \in S} \frac{\|\rho(s) v-v\|^{2}}{\|v\|^{2}}=\inf _{\rho \in \Gamma_{0}^{*} 0 \neq v \in \mathcal{H}_{\rho}} \frac{\left\|\frac{1}{S \mid} \sum_{s \in S} \rho(s) v-v\right\|}{\|v\|},
$$

where $\Gamma_{0}^{*}$ denote the space of unitary representations of $\Gamma$ with no invariant non-zero vectors.

Let us now describe the structure of this paper. Section 2 is devoted to a proof of Theorem 1.6. In subsection 3.1 we show that if $n>1$, then the cohomology group of the group extensions of $F_{p}\left[C_{p}\right]$ by $C_{p^{n}}$ is not trivial. Moreover, we prove the existence of "good orbits" in $F_{p}\left[C_{p}\right]$ under the action of $C_{p^{n}}$, and as a result we obtain Corollary 1.7. In subsection 3.3. we prove Corollary 1.9, and in subsection 3.4 we study the structure of the group of tame automorphisms of the free nilpotent group and calculate explicitly its Kazhdan constants. 


\section{Proof of Theorem 1.6}

Let $(\rho, \mathcal{H})$ be a non-trivial unitary irreducible representation of $\Gamma$ and let

$$
W_{0}=\{w \in \mathcal{H} \mid \rho(a) w=w \quad \forall a \in A\}
$$

be the space of $A$-invariant vectors. Since $A \triangleleft \Gamma, W_{0}$ is $\Gamma$ invariant. As $\rho$ is irreducible, we have either $W_{0}=\mathcal{H}$ or $W_{0}=\{0\}$.

If $W_{0}=\mathcal{H}$ then $\rho$ gives rise to a representation $\bar{\rho}$ of $H=\Gamma / A$. It is clear that $\bar{\rho}$ has no invariant vectors and thus for every $v \in \mathcal{H}$ with $\|v\|=1$, we get

$$
\frac{1}{|S|+|B|} \sum_{s \in S}\|\rho(s) v-v\|^{2}=\frac{1}{|S|+|B|} \sum_{s \in S}\|\bar{\rho}(s) v-v\|^{2}>\frac{\epsilon_{H}|S|}{|S|+|B|} .
$$

We assume from now on that $W_{0}=\{0\}$. The restriction of $\rho$ to $A$ decomposes into irreducible representations:

$$
\rho=\oplus_{i=1}^{n} m_{i} \rho_{i}
$$

where each $\rho_{i}$ is one dimensional and the $m_{i}$ are non negative integers. For each $i \in\{1, \ldots, n\}$, we denote the isotopic component $m_{i} \rho_{i}$ by $\chi_{i}$ and we write $X=\left\{\chi_{i}\right\}_{i=1}^{n}$. Then $X$ is in one to one correspondence with a subset of the set of irreducible characters of $A$. By the irreducibility of $\rho, H$ acts transitively on $X$ (by conjugation). This action gives us a representation $\Phi$ of $H$ on $\ell_{2}(X)$ (the vector space of all complex valued functions on $X$ endowed with the standard inner product) defined by $(\Phi(h) F)(\chi)=F\left(\chi^{h}\right)$.

Now assume that for some $\epsilon$ which will be determined later, there exists a unit vector $v \in \mathcal{H}$, s.t

$$
\frac{1}{|S|+|B|} \sum_{g \in \tilde{S} \cup B}\|\rho(g) v-v\|^{2} \leq \epsilon .
$$

Decompose $v$ into its isotopic components: $v=\sum_{\chi \in X} v_{\chi}$. We define $F=F_{v} \in \ell_{2}(X)$ by $F(\chi)=\left\|v_{\chi}\right\|$. Note that $F$ is a unit vector.

The following lemma is a general property of Kazhdan groups.

Lemma 2.1. Let $\Gamma$ be a group generated by a set $S$ with a Kazhdan constant $\epsilon>0$ with respect to $S$. Let $0<\delta<\epsilon$ be given. Let $(\rho, \mathcal{H})$ be a unitary representation of $\Gamma$ having a unit vector $v \in \mathcal{H}$, satisfying $\frac{1}{|S|} \sum_{s \in S}\|\rho(s) v-v\|^{2} \leq \delta$. Then there exists an invariant vector $v_{0}$ satisfying $\left\|v-v_{0}\right\|^{2}<\frac{\delta}{\epsilon}$.

Proof. Decompose $\rho$ into its trivial and non-trivial components, $\rho=\sigma_{0}+\sigma_{1}$, and accordingly decompose $v=v_{0}+v_{1}$.

Now $\sigma_{1}$ has no invariant vectors, therefore 


$$
\delta \geq \frac{1}{|S|} \sum_{s \in S}\|\rho(s) v-v\|^{2}=\frac{1}{|S|} \sum_{s \in S}\left\|\sigma_{1}(s) v_{1}-v_{1}\right\|^{2}>\epsilon\left\|v_{1}\right\|^{2} .
$$

This implies that $\left\|v_{1}\right\|^{2}<\frac{\delta}{\epsilon}$, hence we get $\left\|v-v_{0}\right\|^{2}<\frac{\delta}{\epsilon}$ as required.

Here is a technical lemma which we will use latter in the proof.

Lemma 2.2. Let $\vec{a}=\left(a_{1}, \ldots, a_{n}\right) \in \mathbb{R}^{n}$ be a unit vector and $\vec{b}=\left(b_{1}, \ldots, b_{n}\right) \in \mathbb{R}^{n}$. Assume that there is some $K \in \mathbb{R}$ such that $\left|b_{i}\right| \leq K / \sqrt{n}$. Then

$$
\sum_{i=1}^{n}\left|a_{i}^{2}-b_{i}^{2}\right| \leq \sum_{i=1}^{n}\left(a_{i}-b_{i}\right)^{2}+2 K \sqrt{\sum_{i=1}^{n}\left(a_{i}-b_{i}\right)^{2}} .
$$

Proof.

$$
\begin{gathered}
\sum_{i=1}^{n}\left(\left|a_{i}^{2}-b_{i}^{2}\right|-\left(a_{i}-b_{i}\right)^{2}\right)=\sum_{\substack{i=1 \\
a_{i} \geq b_{i}}}^{n}\left(\left(a_{i}^{2}-b_{i}^{2}\right)-\left(a_{i}-b_{i}\right)^{2}\right)+\sum_{\substack{i=1 \\
b_{i}>a_{i}}}^{n}\left(\left(b_{i}^{2}-a_{i}^{2}\right)-\left(a_{i}-b_{i}\right)^{2}\right)= \\
\sum_{\substack{i=1 \\
a_{i} \geq b_{i}}}^{n} 2 b_{i}\left(a_{i}-b_{i}\right)+\sum_{\substack{i=1 \\
b_{i}>a_{i}}}^{n} 2 a_{i}\left(b_{i}-a_{i}\right) \leq \frac{2 K}{\sqrt{n}} \sum_{\substack{i=1 \\
a_{i} \geq b_{i}}}^{n}\left(a_{i}-b_{i}\right)+\frac{2 K}{\sqrt{n}} \sum_{\substack{i=1 \\
b_{i}>a_{i}}}^{n}\left(b_{i}-a_{i}\right) \\
=\frac{2 K}{\sqrt{n}} \sum_{i=1}^{n}\left|a_{i}-b_{i}\right| \leq 2 K \sqrt{\sum_{i=1}^{n}\left(a_{i}-b_{i}\right)^{2}} .
\end{gathered}
$$

The last inequality is by the Cauchy Schwarz inequality.

Lemma 2.3. $\frac{1}{|S|} \sum_{s \in S}\|\Phi(s) F-F\|^{2} \leq \frac{\epsilon(|S|+|B|)}{|S|}$.

Proof. From the definition of $\Phi$, we have:

$$
\begin{gathered}
\frac{1}{|S|+|B|} \sum_{s \in S}\|\Phi(s) F-F\|^{2}=\frac{1}{|S|+|B|} \sum_{s \in S} \sum_{\chi \in X}\left|F\left(\chi^{s}\right)-F(\chi)\right|^{2}= \\
\frac{1}{|S|+|B|} \sum_{s \in S} \sum_{\chi \in X}\left|\left(\left\|v_{\chi^{s}}\right\|-\left\|v_{\chi}\right\|\right)\right|^{2}=\frac{1}{|S|+|B|} \sum_{s \in S} \sum_{\chi \in X}\left|\left(\left\|v_{\chi^{s}}\right\|-\left\|\rho(s) v_{\chi}\right\|\right)\right|^{2} \\
\leq \frac{1}{|S|+|B|} \sum_{s \in S} \sum_{\chi \in X}\left\|\rho(s) v_{\chi}-v_{\chi^{s}}\right\|^{2} \leq \epsilon .
\end{gathered}
$$


Denote by $c=\frac{|B|}{|S|}$, now by Lemma 2.1 we obtain that there exists an invariant (i.e. constant) function $G$ such that $\|F-G\|^{2}<\frac{(1+c) \cdot \epsilon}{\epsilon_{H}}$. Thus

$$
\|G\|=K<\|F\|+\sqrt{\frac{(1+c) \cdot \epsilon}{\epsilon_{H}}}=1+\sqrt{\frac{(1+c) \cdot \epsilon}{\epsilon_{H}}}
$$

and so $G(\chi)=K / \sqrt{|X|}$. We choose $u \in \mathcal{H}$ such that for each $\chi \in X$,

$$
G(\chi)=\left\|u_{\chi}\right\|
$$

Since $W_{0}=\{0\}$, the following holds:

$$
\begin{gathered}
\epsilon_{A}<\frac{1}{|B||H|} \sum_{b \in B} \sum_{h \in H}\left\|\rho\left(b^{h}\right) v-v\right\|^{2}=\frac{1}{|B||H|} \sum_{b \in B} \sum_{h \in H} \sum_{\chi \in X}\left\|\chi\left(b^{h}\right) v_{\chi}-v_{\chi}\right\|^{2}= \\
\frac{1}{|B||H|} \sum_{b \in B} \sum_{h \in H} \sum_{\chi \in X}\left|\chi^{h}(b)-1\right|^{2}\left\|v_{\chi}\right\|^{2}=\frac{1}{|B||H|} \sum_{b \in B} \sum_{h \in H} \sum_{\chi \in X}|\chi(b)-1|^{2} F\left(\chi^{h^{-1}}\right)^{2} .
\end{gathered}
$$

Now,

$$
\begin{gathered}
\left|\frac{1}{|B||H|} \sum_{b \in B} \sum_{h \in H} \sum_{\chi \in X}\right| \chi(b)-\left.1\right|^{2} F\left(\chi^{h^{-1}}\right)^{2}-\frac{1}{|B||H|} \sum_{b \in B} \sum_{h \in H} \sum_{\chi \in X}|\chi(b)-1|^{2} G(\chi)^{2} \mid \leq \\
\frac{1}{|B||H|} \sum_{b \in B} \sum_{h \in H} \sum_{\chi \in X}|\chi(b)-1|^{2}\left|F\left(\chi^{h^{-1}}\right)^{2}-G(\chi)^{2}\right| \leq
\end{gathered}
$$

$4 \cdot \frac{1}{|B||H|} \sum_{b \in B} \sum_{h \in H} \sum_{\chi \in X}\left|F\left(\chi^{h^{-1}}\right)^{2}-G(\chi)^{2}\right|=4 \cdot \frac{1}{|B||H|} \sum_{b \in B} \sum_{h \in H} \sum_{\chi \in X}\left|F\left(\chi^{h}\right)^{2}-G(\chi)^{2}\right|$.

Now, substituting $a=F$ and $b=G$ in Lemma 2.2, we get:

$$
\begin{gathered}
4 \cdot \sum_{\chi \in X}\left|F\left(\chi^{h}\right)^{2}-G(\chi)^{2}\right| \leq 4 \cdot\left(\sum_{\chi \in X}\left(F\left(\chi^{h}\right)-G(\chi)\right)^{2}+2 K \sqrt{\sum_{\chi \in X}\left(F\left(\chi^{h}\right)-G(\chi)\right)^{2}}\right)= \\
4 \cdot\left(\sum_{\chi \in X}(F(\chi)-G(\chi))^{2}+2 K \sqrt{\sum_{\chi \in X}(F(\chi)-G(\chi))^{2}}\right) \leq 4 \cdot\left(\|F-G\|^{2}+2 K \sqrt{\|F-G\|^{2}}\right)
\end{gathered}
$$




$$
<4 \cdot\left(\frac{(1+c) \cdot \epsilon}{\epsilon_{H}}+2\left(1+\sqrt{\frac{(1+c) \cdot \epsilon}{\epsilon_{H}}}\right) \sqrt{\frac{(1+c) \cdot \epsilon}{\epsilon_{H}}}\right)=12 \cdot \frac{(1+c) \cdot \epsilon}{\epsilon_{H}}+8 \cdot \sqrt{\frac{(1+c) \cdot \epsilon}{\epsilon_{H}}}
$$

Now

$$
\begin{gathered}
\epsilon_{A}<\frac{1}{|B||H|} \sum_{b \in B} \sum_{h \in H}\left\|\rho\left(b^{h}\right) v-v\right\|^{2}=\frac{1}{|B||H|} \sum_{b \in B} \sum_{h \in H} \sum_{\chi \in X}|\chi(b)-1|^{2}\left\|v_{\chi^{h}}\right\|^{2} \leq \\
12 \cdot \frac{(1+c) \cdot \epsilon}{\epsilon_{H}}+8 \sqrt{\frac{(1+c)^{2} \cdot \epsilon}{\epsilon_{H}}}+\frac{1}{|B||H|} \sum_{b \in B} \sum_{h \in H} \sum_{\chi \in X}|\chi(b)-1|^{2} G(\chi)^{2} \leq \\
12 \cdot \frac{(1+c) \cdot \epsilon}{\epsilon_{H}}+8 \sqrt{\frac{(1+c) \cdot \epsilon}{\epsilon_{H}}}+12 \cdot \frac{(1+c) \cdot \epsilon}{\epsilon_{H}}+8 \sqrt{\frac{(1+c) \cdot \epsilon}{\epsilon_{H}}}+\frac{1}{|B||H|} \sum_{b \in B} \sum_{h \in H} \sum_{\chi \in X}|\chi(b)-1|^{2}\left\|v_{\chi}\right\|^{2}= \\
24 \cdot \frac{(1+c) \cdot \epsilon}{\epsilon_{H}}+16 \sqrt{\frac{(1+c) \cdot \epsilon}{\epsilon_{H}}}+\frac{1}{|B||H|} \sum_{b \in B} \sum_{h \in H} \sum_{\chi \in X}|\chi(b)-1|^{2}\left\|v_{\chi}\right\|^{2}= \\
24 \cdot \frac{(1+c) \cdot \epsilon}{\epsilon_{H}}+16 \cdot \frac{(1+c) \cdot \epsilon}{\epsilon_{H}}+\frac{1}{|B|} \sum_{b \in B} \sum_{\chi \in X}|\chi(b)-1|^{2}\left\|v_{\chi}\right\|^{2}< \\
24 \cdot \frac{(1+c) \cdot \epsilon}{\epsilon_{H}}+16 \sqrt{\frac{(1+c) \cdot \epsilon}{\epsilon_{H}}}+\left(1+\frac{1}{c}\right) \epsilon .
\end{gathered}
$$

If we choose

$$
\epsilon \leq \frac{\epsilon_{H} \epsilon_{A}}{512 \cdot\left(1+c+\frac{1}{c}\right)}
$$

we get a contradiction (the last inequality follows from the fact that $\epsilon, \epsilon_{H} \leq 1$ ) and this finishes the proof of Theorem 1.6.

\section{Applications}

\subsection{Solvable Groups}

Lubotzky and Weiss ([LW], Corollary 3.3) proved that Kazhdan constants of an infinite family of solvable groups with bounded derived length, with respect to any bounded generating set 
are going to zero. The general way to give a positive Kazhdan constant for an infinite family of solvable groups is by using Alon-Roichman result which require a generating set of logarithmic size of the group.

The first improvement for some solvable groups is due to Meshulam and Wigderson [MW] (based on [ALW]). They constructs a family of solvable groups of the form $G_{i+1}=G_{i} \ltimes \mathbb{F}_{p_{i}}\left[G_{i}\right]$ where $G_{i}$ is a finite group, $p_{i}$ is a prime and $G_{i+1}$ is the natural semi-direct product.

Theorem 3.1. ([MW], Theorem 1.7) There exists a group $G_{1}$ and a sequence of primes $p_{i}$, such that for any $0<\epsilon<1$ we have

$$
g_{\epsilon}\left(G_{n}\right) \leq \log ^{\left(n-\log ^{*} n\right)}\left|G_{n}\right|
$$

where $\log ^{k}$ denotes the $k$ times iterated logarithm function.

Influenced by Meshulam and Wigderson methods, we will give in this section a construction of a family of meta-abelian groups $\Gamma_{p}$ (split and non-split), with a substantial improvement for $g_{\epsilon}\left(\Gamma_{p}\right)$ (compared to Alon-Roichman).

\subsection{Meta Abelian Groups}

\subsubsection{Group Cohomology and Non-Splitting Extension}

Definition 3.2. If $\pi: \Gamma \rightarrow H$ is surjective homomorphism of groups, then a lifting of $x \in H$ is an element $l(x) \in \Gamma$ with $\pi(l(x))=x$.

Let $A$ be an abelian group, $H$ a group with a homomorphism

$$
\theta: H \rightarrow \operatorname{Aut}(A)
$$

Let $H^{2}(H, A, \theta)$ be the second cohomology group. As is well known (see [Rot], ch. 7), $H^{2}(H, A, \theta)$ parameterizes groups extension of $A$ by $H$ with respect to $\theta$.

Let $p$ be a prime, $2 \leq n \in \mathbb{N}$ and let $C_{p}, C_{p^{n}}$ be the cyclic groups of order $p$ and $p^{n}$ respectively. Let $\mathbb{F}_{p}$ be the field of order $p$ and $\mathbb{F}_{p}\left[C_{p}\right]$ the group algebra. Every element $f \in \mathbb{F}_{p}\left[C_{p}\right]$ can be represented as $f=\sum_{x \in C_{p}} f(x) x$.

Let $\theta: C_{p^{n}} \rightarrow \operatorname{Aut}\left(\mathbb{F}_{p}\left[C_{p}\right]\right)$ be the left translation $(\bmod p)$ homomorphism.

Lemma 3.3. For every $n \geq 2$

$$
H^{2}\left(C_{p^{n}}, \mathbb{F}_{p}\left[C_{p}\right], \theta\right) \neq 0
$$

hence, there is a non-split extension of $A=\mathbb{F}_{p}\left[C_{p}\right]$ by $H=C_{p^{n}}$. 
Proof. Let $\tilde{\Gamma}=C_{p^{n+1}} \ltimes \mathbb{F}_{p}\left[C_{p}\right]$, and let $z$ be a generator of $C_{p^{n+1}}$.

Let $0 \neq a \in \mathbb{F}_{p}$ and define $\alpha=\sum_{x \in C_{p}} a x \in \mathbb{F}_{p}\left[C_{p}\right]$ (embedded in $\tilde{\Gamma}$ ), it is easy to verify that the subgroup generated by $z^{-p^{n}} \alpha$ is a normal subgroup of order $p$. Put

$$
\Gamma=\tilde{\Gamma} /<z^{-p^{n}} \alpha>
$$

and define a homomorphism $\varphi: \tilde{\Gamma} \rightarrow C_{p^{n}}$ by setting $\varphi(z)=y$ where $y$ is a generator of $C_{p^{n}}$, and $\varphi(\beta)=1$ for every $\beta \in \mathbb{F}_{p}\left[C_{p}\right]$ (embed in $\tilde{\Gamma}$ ). Now since $\varphi\left(z^{-p^{n}} \alpha\right)=1, \varphi$ factors through $\Gamma$, thus $\Gamma$ is a non-trivial extension of $p$-elementary abelian group of rank $p$ by $C_{p^{n}}$. We claim that $\Gamma$ is a non-split extension of $\mathbb{F}_{p}\left[C_{p}\right]$ by $C_{p^{n}}$. It suffices to show that there is no lifting of $y$ in $\Gamma$ of order $p^{n}$. Let $\beta \in \mathbb{F}_{p}\left[C_{p}\right]$ (embed in $\tilde{\Gamma} /<z^{-p^{n}} \alpha>$ ), the element $z \beta$ satisfy

$$
(z \beta)^{p^{n}}=z^{p^{n}} \cdot \beta^{z^{p^{n}-1}} \cdots \beta^{z^{2}} \cdot \beta^{z} \cdot \beta
$$

Note that all the elements in the right side belong to the abelian subgroup $\mathbb{F}_{p}\left[C_{p}\right]$. One can check that

$$
\beta^{z^{p^{n}-1}} \cdots \beta^{z^{2}} \cdot \beta^{z} \cdot \beta=p^{n-1} \cdot \sum_{x \in C_{p}}\left(\sum_{x^{\prime} \in C_{p}} \beta\left(x^{\prime}\right)\right) x=0 .
$$

Hence for every $\beta \in \mathbb{F}_{p}\left[C_{p}\right]$, we get $(z \beta)^{p^{n}}=z^{p^{n}}=\alpha \neq 0$ and the result follow.

\subsubsection{Proof of Corollary 1.7;}

We begin by proving that for any $0<\epsilon<1$, there exist a subset $B \subseteq \mathbb{F}_{p}\left[C_{p}\right]$ such that the average Kazhdan constant of $\mathbb{F}_{p}\left[C_{p}\right]$ with respect to $\tilde{B}=B^{C_{p}}$ is greater than $\epsilon$.

Given $f, g \in \mathbb{F}_{p}\left[C_{p}\right]$, define the product $f \cdot g=\sum_{x \in C_{p}} f(x) g(x) \in \mathbb{F}_{p}$. Let $e_{p}(\alpha)=\exp \left(\frac{2 \pi \alpha i}{p}\right)$.

A multiset $\tilde{B} \subset \mathbb{F}_{p}\left[C_{p}\right]$ is called $\delta$-balanced if for all $0 \neq f \in \mathbb{F}_{p}\left[C_{p}\right]$

$$
\left|\sum_{h \in \tilde{B}} e_{p}(f \cdot h)\right| \leq(1-\delta)|\tilde{B}| .
$$

If $\tilde{B}$ is $\delta$-balanced then

$$
\begin{gathered}
1-\left(\frac{1}{\left|\tilde{B} \cup \tilde{B}^{-1}\right|}\left|\sum_{h \in \tilde{B} \cup \tilde{B}^{-1}} e_{p}(f \cdot h)\right|\right)=1-\frac{1}{2|\tilde{B}|}\left|\sum_{h \in \tilde{B}} e_{p}(f \cdot h)+\sum_{h \in \tilde{B}^{-1}} e_{p}(f \cdot h)\right| \geq \\
1-\frac{1}{2|\tilde{B}|}\left(\left|\sum_{h \in \tilde{B}} e_{p}(f \cdot h)\right|+\left|\sum_{h \in \tilde{B}^{-1}} e_{p}(f \cdot h)\right|\right)=1-\frac{1}{|\tilde{B}|}\left|\sum_{h \in \tilde{B}} e_{p}(f \cdot h)\right| \geq \delta .
\end{gathered}
$$


Since $\mathbb{F}_{p_{\tilde{B}}}\left[C_{p}\right]$ is an abelian group, it is easy to check that the spectral gap of $\mathbb{F}_{p}\left[C_{p}\right]$ with respect to $\tilde{B} \cup \tilde{B}^{-1}$ is:

$$
\min _{\chi \neq 1}\left|\frac{1}{\left|\tilde{B} \cup \tilde{B}^{-1}\right|} \sum_{h \in \tilde{B} \cup \tilde{B}^{-1}} \chi(h)-1\right| .
$$

So, from the discussion in subsection 1.2, we obtain that if $\tilde{B}$ is $\delta$-balanced, then the average Kazhdan constant of $A$ with respect to $\tilde{B} \cup \tilde{B}^{-1}$ is at least $2 \delta$.

For $f \in \mathbb{F}_{p}\left[C_{p}\right], s \in \mathbb{N}$ and $\delta>0$ let

$$
B_{\delta}(f)=\left\{\left(h_{1}, \ldots, h_{s}\right) \in \mathbb{F}_{p}\left[C_{p}\right]^{s}:\left|\frac{1}{s p} \sum_{i=1}^{s} \sum_{\sigma \in C_{p}} e_{p}\left(\sigma h_{i} \cdot f\right)\right|>1-\delta\right\} .
$$

For $f \in \mathbb{F}_{p}\left[C_{p}\right]$, define a linear map $T_{f}: \mathbb{F}_{p}\left[C_{p}\right] \rightarrow \mathbb{F}_{p}\left[C_{p}\right]$ by $T_{f}(h)=h f$. Denote by $C_{p} f$ the orbit of $f$ under the action of $C_{p}$. It is clear that

$$
\operatorname{dim}\left(\operatorname{Span}\left\{C_{p} f\right\}\right)=\operatorname{rank} T_{f}
$$

Let

$$
V_{r}\left(\mathbb{F}_{p}\right)=\left\{f \in \mathbb{F}_{p}\left[C_{p}\right]: \operatorname{rank} T_{f}=r\right\} .
$$

Let $C_{p^{n}}$ be the cyclic group of order $p^{n}$ for some $n \in \mathbb{N}$. We defined above the following representation of $C_{p^{n}}$ :

$$
\theta: C_{p^{n}} \rightarrow \operatorname{Aut}\left(\mathbb{F}_{p}\left[C_{p}\right]\right)
$$

Let $a$ be a generator of $C_{p^{n}}$, set $\alpha=\theta(a)$, then $\alpha$ satisfy $\alpha^{p^{n}}=1$. Thus $\alpha$ is a root of the polynomial $x^{p^{n}}-1=(x-1)^{p^{n}}$. So the minimum polynomial of $\alpha$ is $(x-1)^{k}$ for some $k \leq p^{n}$. Hence, 1 is the only eigenvalue of $\alpha$, so the trivial representation (which is the constant vectors) is the only irreducible representation of $C_{p^{n}}$.

Now it is immediate to check that $\mathbb{F}_{p}\left[C_{p}\right]$ is an indecomposable representation of dimension $p$. Furthermore, consider the Jordan canonical form of $\alpha$, we see that a subspace of $\mathbb{F}_{p}\left[C_{p}\right]$ is indecomposable if and only if it correspond to a Jordan block which may be of size $1 \leq s \leq p$. Therefore, if one represent $\alpha$ in it Jordan canonical from, then we get that for every $1 \leq k \leq p$ the number of elements in $\mathbb{F}_{p}\left[C_{p}\right]$ of rank $k$, is $(p-1) p^{k-1}$.

Meshulam and Wigderson (see Proposition 3.2 in [MW]) proved the following:

Proposition 3.4. If $\operatorname{rank} T_{f}=r$ then

$$
\operatorname{Prob}\left(B_{\delta}(f)\right) \leq 8 \exp \left(\frac{-(1-2 \delta)^{2} r s}{4}\right) .
$$

The following proposition is a variant of Theorem 1.2 in [MW]. 
Proposition 3.5. For any $0<\delta<\frac{1}{2}$ there exist $s=O\left(\frac{1}{(1-2 \delta)^{2}} \cdot \ln p\right)$ elements $h_{1}, \ldots, h_{s} \in$ $\mathbb{F}_{p}\left[C_{p}\right]$ such that the multiset $\tilde{B}=\bigcup_{i=1}^{s} C_{p} h_{i} \subset \mathbb{F}_{p}\left[C_{p}\right]$ is $\delta$-balanced and the average Kazhdan constant of $\mathbb{F}_{p}\left[C_{p}\right]$ with respect to $\tilde{B} \cup \tilde{B}^{-1}$ is $2 \delta$.

Proof.

$$
\begin{gathered}
\operatorname{Pr}\left(\bigcup_{0 \neq f \in \mathbb{F}_{p}\left[C_{p}\right]} B_{\delta}(f) \leq \sum_{0 \neq f \in \mathbb{F}_{p}\left[C_{p}\right]} B_{\delta}(f) \leq\right. \\
8 \sum_{r \geq 1}\left|V_{r}\left(\mathbb{F}_{p}\right)\right| \exp \left(\frac{-(1-2 \delta)^{2} r s}{4}\right) \leq \\
8 \sum_{r=1}^{p} p^{r} \exp \left(\frac{-(1-2 \delta)^{2} r s}{4}\right)=8 \sum_{r=1}^{p}\left(p^{4} \exp \left(-(1-2 \delta)^{2} s\right)^{\frac{r}{4}}=\right. \\
8\left[p^{4} \exp \left(-(1-2 \delta)^{2} s\right)\right]^{\frac{1}{4}} \frac{1-\left[p^{4} \exp \left(-(1-2 \delta)^{2} s\right]^{\frac{p}{4}}\right.}{1-\left[p^{4} \exp \left(-(1-2 \delta)^{2} s\right]^{\frac{1}{4}}\right.}<1
\end{gathered}
$$

For any $c>1$ and for $p$ large enough, if one choose $s=\frac{4}{(1-2 \delta)^{2}}(c \ln p)$, then the probability that $h_{1}, \ldots, h_{s}$ is not $\delta$-balanced is strictly less than 1 and the result follow.

The last proposition still holds if we replace the group $C_{p}$ by $C_{p^{n}}$ with the action by left translation $\bmod p$ on $\mathbb{F}_{p}\left[C_{p}\right]$. This follow easily from the fact that for every $f \in \mathbb{F}_{p}\left[C_{p}\right]$, every element in the multiset $C_{p} f$ appears $p^{n-1}$ times in the multiset $C_{p^{n}} f$.

Now we are ready to complete the proof of Corollary 1.7. By AR for any $0<\epsilon_{1}<1$, there is a generating set $S$ for $C_{p^{n}}$ of size $O_{\epsilon_{1}}\left(\log \left|C_{p^{n}}\right|\right)$ such that the Kazhdan constant of $C_{p^{n}}$ with respect to $S$ is bigger than $\epsilon_{1}$. Let $\Gamma$ be any extension of $\mathbb{F}_{p}\left[C_{p}\right]$ by $C_{p^{n}}$ with respect to to the action of left translation. Let $\tilde{S}$ be any lifting of $S$ to $\Gamma$, from Proposition 3.5 (and the comment afterward), we get that for any $0<\epsilon_{2}<1$ there exist a set $B \subset \mathbb{F}_{p}\left[C_{p}\right]$ such that the Kazhdan constant of $\mathbb{F}_{p}\left[C_{p}\right]$ with respect to $\tilde{B}=B^{C_{p^{n}}}$ is greater then $\epsilon_{2}$. Now from Theorem 1.6 the proof is complete.

\section{$3.3 \quad p$-Groups}

Fix a prime $p$, for any group $\Gamma$, define $\Gamma^{p}$ to be the subgroup generated by $\left\{g^{p}: g \in \Gamma\right\}$. The lower $p$-series (also called the lower central $p$-series or the lower exponent- $p$ series) of $\Gamma$ is the descending series

$$
\Gamma=\varphi_{1}(\Gamma) \geq \varphi_{2}(\Gamma) \geq \cdots \geq \varphi_{n}(\Gamma) \geq \cdots
$$

defined by

$$
\varphi_{i+1}(\Gamma)=\varphi_{i}(\Gamma)^{p}\left[\varphi_{i}(\Gamma), \Gamma\right]
$$


for $i \geq 1$. The group $\Gamma$ is said to have class $n$, if $\varphi_{n}(\Gamma)$ is the last non-identity element of the lower $p$-series.

This subsection uses results and properties of the lower $p$-series. Proofs of the results can be found in Huppert and Blackburn [HB] (ch. VIII) and in Blackburn, et el. [BNV] (ch. 4). On can check the following:

Proposition 3.6. For all positive integer $i, \varphi_{i+1}(\Gamma)$ is the smallest normal subgroup of $\Gamma$ lying in $\varphi_{i}(\Gamma)$, such that $\varphi_{i}(\Gamma) / \varphi_{i+1}(\Gamma)$ is an elementary abelian $p$ group and is central in $\Gamma / \varphi_{i+1}(\Gamma)$.

Let $F_{k}$ be the free group on $k$ generators. Let $\Gamma_{k, n}=F_{k} / \varphi_{n+1}\left(F_{k}\right)$. This group is a $p$-group, called the relatively free group on $k$ generators in the variety of $p$-groups with a lower- $p$ series of class $n$ (for an introduction to variety of a groups see ch. 1 in [Ne]). The dimensions of $\varphi_{i}\left(F_{k}\right) / \varphi_{i+1}\left(F_{k}\right)$ can be calculated explicitly (see [HB]), in particular for the case of $\Gamma_{k, 2}=F_{k} / \varphi_{3}\left(F_{k}\right)$, we have the following lemma:

Lemma 3.7. ([BNV], Lemma 4.2) The Frattini subgroup $\Phi\left(\Gamma_{k, 2}\right)$ of $\Gamma_{k, 2}$ is of order $p^{\frac{1}{2} k(k+1)}$ and index $p^{k}$.

In addition, following the proof of the last Lemma, one can show that the order of the commutator subgroup $\Gamma_{k, 2}^{\prime}$ is $p^{\frac{1}{2} k(k-1)}$.

\subsubsection{Proof of Corollary 1.9;}

Let $\Gamma_{k, 2}$ be the relatively free group in the variety of $p$-groups with a lower- $p$ series of class. The Frattini subgroup of $\Gamma_{k, 2}$ is

$$
\Phi\left(\Gamma_{k, 2}\right)=\Gamma_{k, 2}^{p}\left[\Gamma_{k, 2}, \Gamma_{k, 2}\right]=\varphi_{2}\left(\Gamma_{k, 2}\right) .
$$

Hence if $\tilde{S} \subset \Gamma_{k, 2}$ is such that its image in $\Gamma_{k, 2} / \varphi_{2}\left(\Gamma_{k, 2}\right)$ generates $\Gamma_{k, 2} / \varphi_{2}\left(\Gamma_{k, 2}\right)$, then $\tilde{S}$ generates $\Gamma_{k, 2}$.

From Proposition 3.6, $\varphi_{2}\left(\Gamma_{k, 2}\right)$ is central in $\Gamma_{k, 2}$, so $\Gamma_{k, 2}$ is a central extension of $\Gamma_{k, 2}^{\prime}=$ $\left[\Gamma_{k, 2}, \Gamma_{k, 2}\right]$ by $\Gamma_{k, 2} / \Gamma_{k, 2}^{\prime}$.

By [AR] for any $0<\epsilon<1$ there is a generating set $S$ for $\Gamma_{k, 2} / \Gamma_{k, 2}^{\prime}$ of size $O_{\epsilon}\left(\log \left|\Gamma_{k, 2} / \Gamma_{k, 2}^{\prime}\right|\right)=$ $O_{\epsilon}\left(\log \left|p^{2 k}\right|\right)$ such that the average Kazhdan constant of $\Gamma_{k, 2} / \Gamma_{k, 2}^{\prime}$ with respect to $S$ is bigger than $\epsilon$. Let $\tilde{S}$ be any lifting of $S$ to $\Gamma_{k, 2}$. We claim that the average Kazhdan constant of $\Gamma_{k, 2}$ with respect to $\tilde{S}$ is bigger than $\epsilon / 4$. Indeed, one dimensional representations are trivial on $\Gamma_{k, 2}^{\prime}$ (since it is the commutator subgroup) and hence factor through $\Gamma_{k, 2} / \Gamma_{k, 2}^{\prime}$, and for higher dimensional representations we use theorem 1.8. We have shown that $\Gamma_{k, 2}$ has a generating set of size

$$
O_{\epsilon}\left(\log \left|\Gamma_{k, 2} / \Gamma_{k, 2}^{\prime}\right|\right)=O_{\epsilon}\left(\sqrt{\log \left|\Gamma_{k, 2}\right|}\right)
$$


One can repeat the above methods and improve $g_{\epsilon}\left(\Gamma_{k, n}\right)$ for the relatively free group on $k$ generators in the variety of $p$-groups with a lower- $p$ series of class $n>2$.

\subsection{Kazhdan Constants for the Tame Automorphism Groups of Free Nilpo- tent Groups}

Let $F_{k}$ be the free group on $k$ generators $x_{1}, \ldots, x_{k}$. For any $1 \leq i \neq j \leq k$, let $R_{i, j}^{ \pm}, L_{i, j}^{ \pm}$be the following automorphisms of $F_{k}$ :

$$
\begin{aligned}
& R_{i, j}^{ \pm}\left(x_{i}\right)=x_{i} x_{j}^{ \pm 1} \text { and } R_{i, j}^{ \pm}\left(x_{l}\right)=x_{l} \text { if } l \neq i \\
& L_{i, j}^{ \pm}\left(x_{i}\right)=x_{j}^{ \pm 1} x_{i} \text { and } L_{i, j}^{ \pm}\left(x_{l}\right)=x_{l} \text { if } l \neq i
\end{aligned}
$$

These automorphisms are called Nielsen moves. Let $S$ be the set of Nielsen moves of the free group on $k$ generators, it is easy to check that $|S|=4 k(k-1)$.

Definition 3.8. Let $F_{k}$ be the free group on $k$ generators and $W=\gamma_{c+1}\left(F_{k}\right)$ be the $(c+1)$-th term of the lower central series of $F_{k}$. Then $F_{k} / W$ is called the free nilpotent group of class $c$ and will be denoted by $F_{k}(c)$.

Let $U$ be a characteristic subgroup of $F_{k}$, then it is clear that every automorphism of $F_{k}$ induces an automorphism on $F_{k} / U$. This fact bring us to the following definition.

Definition 3.9. An automorphism of the free nilpotent group $F_{k}(c)$ is said to be tame if it is in the image of the natural projection map $A u t\left(F_{k}\right) \rightarrow \operatorname{Aut}\left(F_{k}(c)\right)$. The subgroup of all the tame automorphisms is denoted by $A_{k}(c)$.

The group $A_{k}(c)$ has the following short exact sequence:

$$
1 \rightarrow K_{1} \rightarrow A_{k}(c) \rightarrow S L_{k}(\mathbb{Z}) \rightarrow 1
$$

where $K_{1}$ is a nilpotent group of class $c-1$ (see And for details).

Lubotzky and Pak showed in [LP] that for $k \geq 3$, the group $A_{k}(c)$ is a lattice in some Lie group which has Kazhdan property $(\mathrm{T})$, hence it has property $(\mathrm{T})$. Their approach does not give an estimate for the Kazhdan constant. Mallahi [MAK] following Burger, Shalom and Kassabov (see [Bur, Sh1, Kas1]), used the spectral measure on the dual group $\hat{\mathbb{Z}}^{m}$ correspond to $S L_{k}(\mathbb{Z})$ and prove that the group $A_{k}(c)$ has relative property $(\mathrm{T})$ with respect to the center of $K_{1}$. Before we state Mallahi results in a more general setting (which follows easily from Mallahi's proof), we give some notation. Let $k, c \in \mathbb{N}$, we define

$$
\delta(k, c)=\frac{2 \sqrt{c}}{\sqrt{(84 \sqrt{k}+1920)(4 k)^{c}}} .
$$


Theorem 3.10. (Theorem 2.5.6 in [MAK]) Let $k \geq 3$ be an integer, $A_{k}(c)$ be the group of tame automorphisms of the free nilpotent group of class $c$ and $S$ the set of Nielsen transformations. Let $A$ be the center of $A_{k}(c)$ and let $Z$ be the center of $K_{1} / A$. Let $(\rho, \mathcal{H})$ be a unitary representation of $A_{k}(c) / A$ with $(S, \delta(k, c))$-invariant vector. Then $\mathcal{H}$ contains a non-zero $Z$-invariant vector.

From the last theorem, Mallahi deduce the following:

Theorem 3.11. (Theorem 2.5.7 in [MAK]) Let $k \geq 3$ be an integer, $A_{k}(c)$ be the group of tame automorphisms of the free nilpotent group of class $c$ and $S$ the set of Nielsen transformations. If $c<2 k+1$, then the Kazhdan constant $\epsilon(k, c)$ of $A_{k}(c)$ with respect to $S$ is greater than or equal to

$$
\frac{\sqrt{c}}{\sqrt{(84 \sqrt{k}+1920)(4 k)^{c}}} .
$$

The limitation for $c<2 k+1$ is based on the following fact due to Formanek (Theorem 6 in $[$ For $]$ :

Theorem 3.12. Let $F_{k}(C)$ be the free nilpotent group of rank $k$ and class $c$ where $k, c \geq 2$. Then the automorphism group has a non-trivial center iff $c=2 k l+1$ for $l \geq 1$.

The goal of this section is to extend Mallahi result and calculate Kazhdan constant of $A_{k}(c)$ for any $k \geq 3$ and $c \geq 1$. Using the same notations as above we prove:

Theorem 3.13. Let $k \geq 3$ be an integer, $A_{k}(c)$ be the group of the tame automorphisms of the free nilpotent group of class $c$ and $S$ the set of Nielsen transformations, then the Kazhdan constant $\epsilon(k, c)$ of $A_{k}(c)$ with respect to $S$ satisfy:

$$
\epsilon(k, c) \geq \begin{cases}\frac{\sqrt{c}}{\sqrt{(84 \sqrt{k}+1920) 4^{c+3} k^{c+4}}} & \text { if } c=2 k l+1 \text { for some } l \in \mathbb{N} ; \\ \frac{\sqrt{c-1}}{\sqrt{(84 \sqrt{k}+1920) 4^{c+3} k^{c+3}}} & \text { if } c=2 k l+2 \text { for some } l \in \mathbb{N} \\ \frac{\sqrt{c-2}}{\sqrt{(84 \sqrt{k}+1920) 4^{c+3} k^{c+2}}} & \text { if } c=2 k l+3 \text { for some } l \in \mathbb{N} \\ \frac{\sqrt{c-3}}{\sqrt{(84 \sqrt{k}+1920) 4^{c+3} k^{c+1}}} & \text { if } c=2 k l+4 \text { for some } l \in \mathbb{N} \\ \frac{\sqrt{c-4}}{\sqrt{(84 \sqrt{k}+1920) 4^{c+3} k^{c}}} & \text { otherwise. }\end{cases}
$$

Proof. For the case $c \leq 2 k$, by Theorem 3.11 we get that the Kazhdan constant of $A_{k}(c)$ with respect to $S$ is

$$
\epsilon(k, c) \geq \frac{\sqrt{c}}{\sqrt{(84 \sqrt{k}+1920)(4 k)^{c}}} .
$$


For the case $c=2 k+1$, denote by $A$ the center of $A_{k}(c)$, by Theorem $[3.12$, $A$ is not trivial. Hence we have the following exact sequence:

$$
1 \rightarrow A \rightarrow A_{k}(c) \rightarrow A_{k}(c) / A \rightarrow 1
$$

and respectively

$$
1 \rightarrow K_{1} / A \rightarrow A_{k}(c) / A \rightarrow S L_{k}(\mathbb{Z}) \rightarrow 1 .
$$

Lemma 3.14. Let $c=2 k+1$ then the Kazhdan constant of $A_{k}(c) / A$ is greater than or equal to $\frac{\delta(k, c)}{2}$.

Proof. Let $(\rho, \mathcal{H})$ be a unitary representation of $A_{k}(c) / A$ and assume that there exist a unit vector $v \in \mathcal{H}$ which is $\left(\frac{\delta(k, c)}{2}, S\right)$-invariant. Let $Z$ be the center of $K_{1} / A$, put

$$
\mathcal{H}_{0}=\{v \in \mathcal{H}: \rho(z) v=v, \forall z \in Z\}
$$

and $\mathcal{H}_{1}=\mathcal{H}_{0}^{\perp}$ (the orthogonal complement). Write $v=v_{0}+v_{1}$ where $v_{i} \in \mathcal{H}_{i}$, by Theorem 3.10 we get that $\mathcal{H}_{0} \neq 0$.

The subspace $\mathcal{H}_{1}$ does not have a $Z$-invariant vector, and therefore it does not have a $(\delta(k, c), S)$-invariant vector. This imply that $\delta(k, c)\left\|v_{1}\right\|<\max _{s \in S}\left\|\rho(s) v_{1}-v_{1}\right\| \leq \frac{\delta(k, c)}{2}$, and we deduce that $\left\|v_{1}\right\|<\frac{1}{2}$.

So $\left\|v_{0}\right\| \geq \frac{1}{2}$ and $\mathcal{H}_{0}$ is $A_{k}(c) / A$-invariant because $Z$ is a normal subgroup. Furthermore, $Z=\gamma_{c}\left(K_{1} / A\right)$, therefore it rise a unitary representation $\bar{\rho}$ of

$$
\left(A_{k}(c) / A\right) / Z=A_{k}(c-1)
$$

on $\mathcal{H}_{0}$.

Let $\tilde{S}$ be the projection of the generators $S$ to $A_{k}(c-1)$, then it is clear that $\tilde{S}$ is the set of Nielsen transformations for $A_{k}(c-1)$. Now, for any $s \in S$ we have

$$
\|\rho(s) v-v\|^{2}=\left\|\bar{\rho}(s) v_{0}-v_{0}\right\|^{2}+\left\|\rho(s) v_{1}-v_{1}\right\|^{2} \leq \frac{\delta(k, c)^{2}}{4}
$$

and therefore

$$
\left\|\bar{\rho}(s) v_{0}-v_{0}\right\|^{2} \leq \frac{\delta(k, c)^{2}}{4} \leq \delta(k, c)^{2}\left\|v_{o}\right\|^{2} \leq \frac{\delta(k, c-1)^{2}}{4}\left\|v_{0}\right\|^{2}
$$

Consequently, the vector $v_{0} \in \mathcal{H}_{0}$ is $\left(\frac{\delta(k, c-1)}{2}, \tilde{S}\right)$-invariant . By Theorem 3.11 we obtain that $\mathcal{H}_{0}$ contains a non-zero invariant vector of $A_{k}(c-1)$ and this vector is also an invariant vector of $A_{k}(c) / A$. 
The group $A_{k}(c)$ doesn't have a non-trivial representation of dimension one, because $A_{k}(c)=\left[A_{k}(c), A_{k}(c)\right]$ (see [Ge]) and $|S|=4 k(k-1)$, therefore from Theorem 1.8 we obtain that the Kazhdan constant $\epsilon(k, c)$ of $A_{k}(c)$ for $c=2 k+1$ with respect to $S$ is greater than or equal to

$$
\frac{\delta(k, c)}{2|S|} \geq \frac{\sqrt{c}}{\sqrt{(84 \sqrt{k}+1920) 4^{c+3} k^{c+4}}} .
$$

For $c>2 k+1$, we continue by induction on $c$ and repeating the same arguments as in Lemma 3.14. Notice that we should take special care for the cases of $2 k l+1 \leq c \leq 2 k l+5$ for any $l \in \mathbb{N}$. Since in case of $c=2 k l+1$ we have a non-trivial center, therefore in those cases we need to choose a Kazhdan constant small enough (as appeared in the theorem) that the induction assumption will be satisfied.

\section{Acknowledgments.}

This paper is part of the author's Ph.D. studies under the guidance of Prof. Alex Lubotzky whom I would like to thank for introducing me the problem and for fruitful conversations. I am grateful to Nir Avni for his assistance in proving Theorem 1.6. I would also like to Thank Eli Bagno, Martin Kassabov and Chen Meiri for insightful discussions.

\section{References}

[ALW] N. Alon, A. Lubotzky and A. Wigderson, Semi-direct Product in Groups and Zig-Zag Proudct in Graphs: Connection and applications. 42nd IEEE Symposium on Foundation of Computer Science (Las Vegas, NY, 2001), 630-637, IEEE Computer Soc. Los Alamitos, CA, 2001.

[And] S. Andreadakis, On the Automorphisms of Free Groups and Free Nilpotent Groups. Proc. London Math. Soc. (3) 151965 239-268.

[AR] N. Alon and Y. Roichman, Random Cayley Graphs and Expanders. Random Structures Algorithms 5 (1994), no. 2, 271-284.

[Bag] E. Bagno, Kazhdan Constants of some Colored Permutation Groups. J. Algebra 282 (2004), no. 1, 205-231.

[BH] R. Bacher and P. de la Harpe, Exact Values of Kazhdan Constants for some Finite Groups. J. Algebra 163 (1994), no. 2, 495-515.

[BHV] B. Bekka, P. de la Harpe and A. Valette, Kazhdan's Property (T). New Mathematical Monographs, 11. Cambridge University Press, Cambridge, 2008. 
[BNV] S. R. Blackburn, P. M. Neumann, and G. Venkataraman, Enumeration of Finite Groups. Cambridge Tracts in Mathematics, 173. Cambridge University Press, Cambridge, 2007.

[Bur] M. Burger, Kazhdan Constants for SL(3, Z). J. Reine Angew. Math. 413 (1991), 36-67.

[For] E. Formanek, Fixed Points and Centers of Automorphism Groups of Free Nilpotent Groups. Comm. Algebra 30 (2002), no. 2, 1033-1038.

[HB] B. Huppert and N. Blackburn, Finite Groups II. Grundlehren der Mathematischen Wissenschaften [Fundamental Principles of Mathematical Sciences], 242. AMD, 44. SpringerVerlag, Berlin-New York, 1982.

[HLW] S. Hoory, N. Linial, and A. Wigderson, Expander Graphs and their Applications. Bull. AMS, 43(2006) 439-561.

[HV] P. de la Harpe and A. Valette, La propriété (T) de Kazhdan pour les Groups Localement Compacts. Astérisque, 175 (1989).

[GZ] T. Gelander and A. Żuk, Dependence of Kazhdan Constants on Generating Subsets. Israel J. Math. 129 (2002), 93-98.

[Ge] S. M. Gersten, A Presentation for the Special Automorphism Group of a Free Group. J. Pure Appl. Algebra 33 (1984), no. 3, 269-279.

[Kas1] M. Kassabov, Universal Lattices and Unbounded Rank Expanders. Invent. Math. 170 (2007), no. 2, 297-326.

[Kas2] M. Kassabov, Kazhdan Constants for $\mathrm{SL}_{n}(\mathbb{Z})$. Internat. J. Algebra Comput. 15 (2005), no. 5-6, 971-995.

[Kaz] D.A. Kazhdan, On the Connection of the Dual Space of a Group with the Structure of its Closed Subgroups. Funk. Anal. Pril. 1 (1967) 71-74.

[KLN] M. Kassabov, A. Lubotzky and N. Nikolov, Finite Simple Groups as Expanders. Proc. Natl. Acad. Sci. USA 103 (2006), no. 16, 6116-6119.

[LR] Z. Landau and A. Russell, Random Cayley Graphs are Expanders: A Simple Proof of the Alon-Roichman Theorem. Electron. J. Combin. 11 (2004), no. 1.

[LS] P. Loh and L. Schulman, Improved Expansion of Random Cayley Graphs. Discrete Mathematics and Theoretical Computer science, 6, (2004), 523-528.

[Lub] A. Lubotzky, Discrete Groups, Expanding Graphs and Invariant Measures. Birkhäuser, 1994. 
[LP] A. Lubotzky and I. Pak, The Product Replacement Algorithm and Kazhdan's Property (T). J. Amer. Math. Soc. 14 (2001), no. 2, 347-363.

[LW] A. Lubotzky and B. Weiss, Groups and Expanders. Expanding graphs (Princeton, NJ, 1992), 95-109, DIMACS Ser. Discrete Math. Theoret. Comput. Sci., 10, Amer. Math. Soc., Providence, RI, 1993.

[MAK] K. Mallahi-Karai, Relative Growth and Kazhdan Property for Arithmetic Groups. $\mathrm{PhD}$ thesis Yale univeristy 2006.

[MW] R. Meshulam and A. Wigderson, Expanders in Group Algebras. Combinatorica 24 (2004), no. 4, 659-680.

[Ne] H. Neumann, Varieties of Groups. Springer-Verlag New York, Inc., New York, 1967.

[PZ] I. Pak and A. Żuk, On Kazhdan Constants and Mixing of Random Walks. Int. Math. Res. Not. (2002), no. 36, 1891-1905.

[Rot] J. J. Rotman, An Introduction to the Theory of Groups. Fourth edition. Graduate Texts in Mathematics, 148. Springer-Verlag, New York, 1995.

[Sh1] Y. Shalom, Bounded Generation and Kazhdan Property (T). Publ. Math. IHES, 90 (1999), 145-168.

Uzy Hadad:

Einstein Institute of Mathematics, The Hebrew University of Jerusalem, Jerusalem 91904, Israel uzy.hadad@gmail.com 